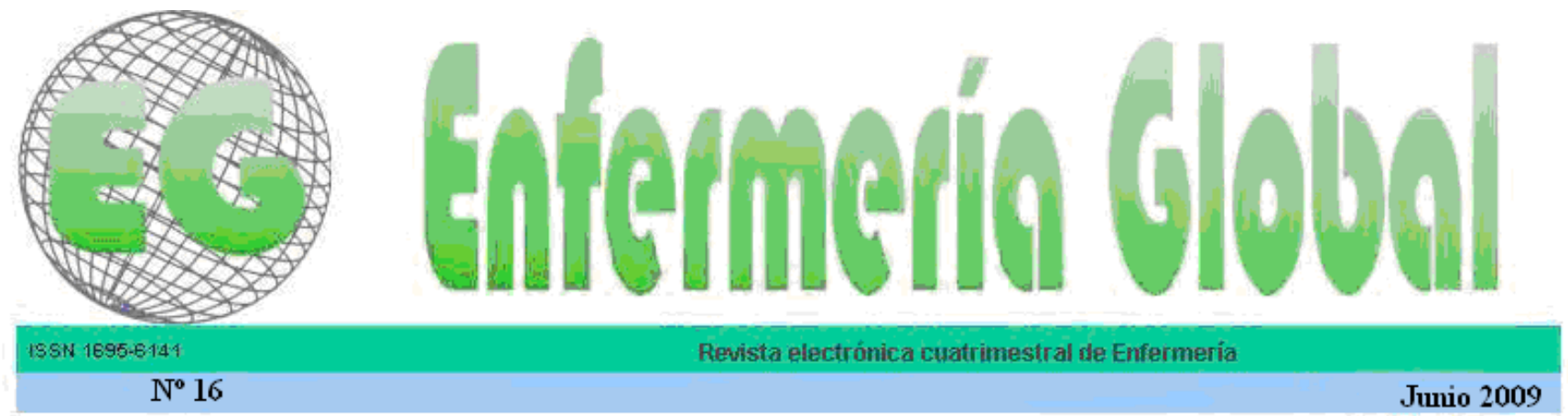

www.um.es/egloball

REVISIONES - RESEYTAS

\title{
APOYO SOCIAL: ELEMENTO CLAVE EN EL AFRONTAMIENTO DE LA ENFERMEDAD CRÓNICA
}

SOCIAL SUPPORT KEY ELEMENT IN CONFRONTING CHRONIC ILLNESS

*Vega Angarita, OM., *González Escobar, DS.

*Magister en Enfermería con énfasis en el cuidado al paciente crónico. Docente asociado, Miembro Grupo Cuidado de Enfermería, Universidad Francisco de Paula Santander. Colombia.

Palabras clave: Enfermedad crónica, cuidadores familiares, apoyo social.

Keywords: Chronic Illness, Family Caregivers, Social Support.

\section{RESUMEN}

La tecnología y los avances en la medicina permiten a las personas sobrevivir a enfermedades fatales y con ello a la incidencia de enfermedades crónicas que se constituyen en principal causa de discapacidad. El apoyo social ha demostrado tener una influencia positiva sobre la experiencia del cuidado de las enfermedades crónicas y de los cuidadores familiares. Se describe como "un proceso de interacción entre personas o grupos de personas, que a través del contacto sistemático establecen vínculos de amistad y de información, recibiendo apoyo material, emocional y afectivo en la solución de situaciones cotidianas en momentos de crisis".

El presente artículo representa la revisión del concepto de apoyo social desde su origen, señala varias definiciones que expertos han dado al tema y cita ejemplos de su aplicación en la investigación con contribuciones de la sociología, psicología, medicina y enfermería. Constituye el marco de referencia del estudio de tipo evaluativo que tiene por objetivo determinar la efectividad del apoyo social dado a través de una red con tecnología punta a cuidadores familiares de personas con enfermedad crónica en Latinoamérica.

\section{ABSTRACT}

Technology and advances in medicine allow people to survive fatal illness and thus, the incidences of chronic illness that are a leading cause of disability. Social support has been shown to have a positive influence on the experience of caring for chronic illness and family caregivers. It is described as "a process of interaction between individuals or groups who through systematic contact establish links of friendship and information, receive material, emotional, and affective support in solving everyday situations in times of crisis." 
This article is to review the concept of social support from its origin, highlighting several definitions that experts have given to the issue and citing examples of its application in research with contributions from Sociology, Psychology, Medicine, and Nursing. It constitutes the reference framework of the assessing study that aims to determine the effectiveness of social support given, through a network with state-of-art technology, to family caregivers of persons with chronic illness in Latin America.

\section{ENFERMEDADES CRÓNICAS DESDE LA PERSPECTIVA SOCIODEMOGRÁFICA Y EPIDEMIOLÓGICA.}

Según informe de la Organización Mundial de la Salud (2008), la carga mundial de enfermedades no trasmisibles sigue aumentando, hacerle frente constituye uno de los principales desafíos para el desarrollo en el siglo XXI. Se calcula que las enfermedades no transmisibles, principalmente las enfermedades cardiovasculares, diabetes, cáncer y enfermedades respiratorias crónicas causaron 35 millones de defunciones en 2005. Esta cifra supone el doble del número de defunciones correspondiente al conjunto de todas las enfermedades infecciosas, incluidos el VIH /SIDA, la tuberculosis y la malaria, enfermedades materno perinatales y carencias nutricionales. El $80 \%$ de las muertes por enfermedades crónicas se registran en países de ingresos bajos y medianos, y aproximadamente 16 millones corresponde a personas de menos de 70 años.

En países como Colombia, las enfermedades crónicas son una causa importante de morbimortalidad y su tendencia es al aumento. Ante esta situación diversos organismos han realizado llamados para la acción; Sin embargo, persiste un bajo reconocimiento por parte de los tomadores de decisiones. Esta realidad se refleja en la ausencia de políticas públicas nacionales para la prevención de las enfermedades crónicas al igual que en la carencia de un sistema de vigilancia que provea información útil y oportuna para la toma de decisiones; panorama que encaja con lo denominado "la epidemia negada de las enfermedades crónicas".

Del total de muertes registradas en Colombia durante 1990 al año 2005 la mortalidad por enfermedades crónicas se incrementó del 59\% al 62.6\%. Las enfermedades crónicas no trasmisibles ocupan el primer puesto entre las principales causas de enfermedad y muerte, sobrepasando incluso las causadas por violencia y accidentes. De estas, la enfermedad cerebrovascular, isquémica del corazón y la hipertensión arterial y sus complicaciones, ocupan el primer lugar, seguido de tumores, enfermedades pulmonares crónicas y enfermedades osteoarticulares degenerativas.

La tecnología y los avances en la medicina permiten a las personas sobrevivir a enfermedades fatales y con ello la incidencia de enfermedades crónicas que constituyen la principal causa de discapacidad, se ha encontrado una estrecha relación entre la prevalencia de enfermedades crónicas no trasmisibles y la discapacidad. Se estima que 500 millones de personas en el mundo, es decir, el 10\% de la población, tiene algún tipo de discapacidad, situación que se incrementa en los países en conflicto, por lo que se calcula que esta cifra podría alcanzar el $18 \%$ de la población total.

Las condiciones que favorecen el aumento de la discapacidad, además del incremento de enfermedades emergentes, reemergentes y crónicas, son el envejecimiento de la población, la desnutrición, los accidentes, el abuso del alcohol y las drogas, la pobreza extrema, las guerras, la violencia social, los desplazamientos de grupos poblacionales y los desastres naturales. De ahí que en Latinoamérica existan aproximadamente 85 millones de personas 
con discapacidad. Probablemente más de un cuarto de la población total de la región se encuentra afectada directa o indirectamente por esta situación, ya sean familiares, amistades o miembros de la comunidad.

En Colombia (DANE, 2005) la situación de la discapacidad guarda correspondencia con la situación mundial, aproximadamente 2.640.000 personas presentan alguna limitación permanente, lo cual equivale al $6.4 \%$ del total de la población colombiana.

En Cúcuta, cuya población se estima en 585.543 habitantes, 71.444 presentan algún tipo de discapacidad, de estos, 5.483 presentan limitaciones para hablar, 6.828 para oír, 4.020 para su autocuidado, 21.091 para ver, 5.290 limitaciones para mover brazos y manos, 4.598 limitaciones para relacionarse con los demás, 10.781 limitaciones para moverse y caminar, 4.983 limitaciones para entender o aprender y 8.370 poseen algún otro tipo de limitación.

\section{IMPACTO DE LA ENFERMEDAD CRÓNICA}

La enfermedad crónica genera en el paciente y su cuidador incertidumbre, desgaste físico, dependencia, cambios en el estilo de vida y tiene repercusiones sobre el área personal y social del individuo.

Según Ponce y Cols., 2007, entre los principales aspectos que relacionan a la familia con la enfermedad crónica, está: a) la familia puede influir en el curso de la enfermedad crónica, entendiendo que la interacción entre la familia y la tipología de la enfermedad pueden tener una influencia positiva o negativa sobre el curso del proceso crónico, y b) la familia como recurso; conviene destacar que la familia es la fuente principal de apoyo social con que cuenta el paciente crónico para afrontar con éxito los problemas a que da lugar la enfermedad, destacando el papel de la cuidadora primaria, que es la que aporta el máximo apoyo instrumental, afectivo y emocional.

En América Latina se ha observado que las personas que tienen un familiar con enfermedad crónica tienen la obligación de desempeñarse como cuidadores, pero no cuentan con la orientación requerida. Se ha documentado de manera importante una deficiente habilidad de cuidado entre estos cuidadores familiares, pero también se han identificado en ellos fortalezas diferentes a las de otras latitudes, que pueden apoyar el servicio requerido como la disponibilidad de soporte social para los cuidadores

Las formas en que los cuidadores afrontan o gestionan las demandas de cuidado depende de lo estresante de la situación y de la etapa de la enfermedad en que se encuentra el receptor de cuidado. Hay una tendencia coherente en los cuidadores a centrar la energía y esfuerzo a hacer frente a los impactos o efectos negativos más que en la solución de problemas y en la búsqueda de beneficios para su salud mental y física. Ante esto, los investigadores han sugerido que el apoyo social reduce las consecuencias negativas de los acontecimientos estresantes que se producen en el cuidado de enfermos crónicos.

Resultados de investigación con enfermedades crónicas consideran que estos beneficios en la población afectada operan como factores de protección. Se ha encontrado que éste protege contra el estrés generado por la enfermedad y capacita al enfermo y su cuidador para reevaluar la situación y adaptarse mejor a ella, ayudándole a desarrollar respuestas de afrontamiento 


\section{DEFINICIONES Y CONCEPTO DEL SOPORTE SOCIAL}

El interés por el concepto de soporte social se origina en los años 70 como una variable primordial en estudios del área de la salud, especialmente en el afrontamiento al estrés en situaciones de crisis. La literatura en relación a los efectos del apoyo social según Broadhead y Kaplan en 1983 proviene de diversas fuentes. En los siguientes párrafos se señalan algunas definiciones que expertos han dado al tema.

Kaplan (1974) lo define en términos de lazos perdurables que desempeñan un papel importante en la integración psicológica y física de una persona. Para Beels, representa los factores existentes en el ambiente y que promueven el curso favorable de la enfermedad. Beigel, Naparstek y Khan (1980) exponen que los individuos se encuentran dentro de una red de relaciones sociales y estas se vuelven importantes cuando proveen el apoyo necesario en situaciones de crisis en la vida en general.

Para Coob (1976), citado por Díaz H, representa la "información dada al sujeto para que crea que es cuidado, amado, estimado y miembro de una red de obligaciones mutuas".

El apoyo social debe entenderse como un concepto de muchos componentes, un conjunto de diferentes significados y explicaciones; es por ello que algunos teóricos prefieren definirlo como un metaconcepto, (López-Cabanas y Chacón, 1997).

De otra parte, también se ha hecho un intento de integración de diferentes explicaciones, recogiendo aquellos aspectos más importantes revelados por otros teóricos. Entre estas definiciones conviene destacar dos:

Para Gottlieb (1998) el apoyo social es una información verbal y no verbal, ayuda tangible o accesibilidad por otros o inferida por su presencia y que tiene efectos conductuales y emocionales beneficiosos en el receptor.

La explicación de Lin y otros (1986) se sustenta en cuatro pilares. Por un lado se puede hablar de la objetividad y de la subjetividad del concepto, diferenciando el apoyo social obtenido y el apoyo social experimentado; ambos son fundamentales para la persona y repercuten sobre su bienestar.

El segundo pilar se centra en la situación contextual del apoyo social y analiza tres áreas en las que podía darse el apoyo social. Desde el punto de vista comunitario, en el macro-nivel, el soporte social permite al individuo sentir que pertenece y está integrado en un sistema social. En un nivel más reducido, de sistemas o redes sociales, el meso-nivel, se ubica en la red de los vínculos personales, en cuyo interior, a menudo indirectamente, se dan afectos o apegos hacia lo demás. En el micro-nivel, en el que se dan vinculaciones muy íntimas y de confianza, se consigue el soporte social no formal, que favorece el nacimiento de una necesidad de compromiso, la persona aguarda reciprocidad en la ayuda sintiéndose en parte responsable por el bienestar de los otros.

Según Saranson (1983), el apoyo social es un término referido a la existencia o disponibilidad de personas con las cuales se puede confiar o contar en periodos de necesidad; quienes proveen al individuo de preocupación, amor y valor personal.

Por su parte Alvarado (1985) lo define como la ayuda importante que proviene de personas o instituciones con las que un sujeto se relaciona y que se actualiza en situaciones de pérdida o de crisis por efecto de estresores psicosociales. 
Barrera (1986) trabajo el concepto, refiriéndose a él como las conexiones que las personas establecen con otros miembros que le son significativos porque le proveen ayuda en tiempos de necesidad, permitiéndole enfrentar situaciones de estrés.

De acuerdo con Muñoz se puede decir que el soporte social es una práctica de cuidado que se da en el intercambio de relaciones entre las personas y se caracteriza por expresiones de afecto, afirmación o respaldo de los comportamientos de otra persona, entrega de ayuda simbólica o material a otra persona. El apoyo social se expresa como la transacción interpersonal que incluye tres aspectos: la expresión de afecto de una persona hacia otra (Apoyo Afectivo), la aprobación de la conducta, pensamientos o puntos de vista de la otra persona (Apoyo Confidencial) y la prestación de ayuda de tipo material (Apoyo Instrumental). A pesar de las diferentes conceptualizaciones de apoyo social existe consenso en que:

El apoyo social posee una estructura que está definida por las características cuantitativas u objetivas de la red de apoyo social, tales como el tamaño, densidad, dispersión geográfica, etc., así como por las características de los contactos y los vínculos sociales que tienen lugar dentro de ella (homogeneidad, reciprocidad o multiplicidad de contactos).

El apoyo social también cuenta con una dimensión funcional donde se analizan los efectos o consecuencias que le producen al sujeto el apoyo, es decir se refiere a la calidad del apoyo social existente y en este sentido se destacan la valoración subjetiva y la disponibilidad de éste (apoyo percibido vs. apoyo recibido) y el que se refiere a los diferentes tipos de apoyo (instrumental, informativo y socioemocional), así como al grado de satisfacción con éste.

El apoyo social como proceso es el que le da una connotación dinámica al constructo, se refiere a los diferentes elementos que influyen en la movilización y la efectividad de éste en el tiempo, lo que está vinculado a su vez por la contextualización del proceso (etapa vital del individuo, tipo de problema al que se enfrenta, condiciones socioeconómicas y culturales).

\section{COMPONENTES Y FUNCIONES DEL SOPORTE SOCIAL.}

El concepto de soporte social y sus operacionalizaciones pueden ser organizadas en tres categorías amplias: redes sociales, soporte social percibido, y soporte social efectivo. La primera se refiere a las conexiones que los individuos tienen para significar a otros en su medio social, teniendo un sentimiento psicológico de comunidad. La segunda representa la valoración cognitiva que hace la persona respecto a quiénes les pueden proveer determinada ayuda en momentos de necesidad. Estas personas no dan soporte social efectivo, pero constituyen para el sujeto personas disponibles o recursos para situaciones problemáticas, lo que le proporciona seguridad dentro y fuera de las situaciones estresantes. Barrera (1986); Cobb (1976); Folkman, Chesney, Johnson y coates (1991). Finalmente, el soporte social efectivo simboliza las acciones soportivas mismas, a la ayuda efectiva, y a las acciones que recibe de los otros en momentos de necesidades. Tardy (1985); Barrera (1986).

Respecto a la función del apoyo social se reconocen cuatro tipos, el emocional que provee al individuo sentimientos de estima, afecto, confianza, seguridad; el valorativo, representa la sensación percibida por el individuo en la que puede contar con alguien, y sentirse acompañado socialmente; el informacional, también conocido como consejo o guía cognitiva, que ayuda a definir y enfrentar los eventos problemáticos mediante asesoramiento por parte de la red; el instrumental constituye la ayuda tangible y material en la solución a 
un problema, Aquí la fuente brinda ayuda práctica y concreta. Pastor, et al. (1994) evaluaron el papel del apoyo social en la experiencia de dolor crónico y obtuvieron que la variable de apoyo social de la red familiar se constituía como predictiva de la ansiedad y depresión así como del dolor en las enfermedades crónicas estudiadas.

La falta de apoyo se relaciona con la aparición de enfermedades físicas (Berkman y Syme, 1979; House et al, 1982; Schwarzer y Leppin, 1992). También se ha relacionado la carencia de apoyo con la aparición de enfermedades mentales como la depresión, la neurosis, se ha visto como las personas que sufren trastornos de esta índole poseen redes sociales pequeñas, menos intercambios de recursos y relaciones no recíprocas. Gottlieb (1988) describe cómo las intervenciones basadas en el apoyo social han tenido tanto auge en la última década. Las razones que enumera se describen a continuación:

- Los efectos positivos que el apoyo social tiene sobre la salud y el bienestar.

- Movilizan recursos informales promoviendo la participación.

- Promueven la validez ecológica y resultan más accesibles.

- Indicadas en una amplia gama de problemas sociales y factores de riesgo físico.

-Aumentan la capacidad de afrontar situaciones de estrés, aumenta la autoresponsabilización y competencias personales.

- Reciben este tipo de apoyo no sólo las personas directamente implicadas sino que afecta positivamente a las personas relacionadas con ellas (cuidadores, familiares, etc.).

De manera general la tendencia ha sido asumir dos posiciones teóricas respecto al papel del apoyo social en la salud. La primera hipótesis sobre los efectos principales o directos, plantea que los efectos de la relación entre apoyo social y salud son independientes del grado de estrés que el individuo experimente ya que el apoyo social aumenta de manera directa y por sí solo el bienestar, la autoestima y la salud; es decir, se establece una relación directamente proporcional entre el apoyo social y la salud, por lo que si se aumenta el apoyo social aumenta la salud, (aunque existe consenso alrededor de este planteamiento, no cuenta con el suficiente fundamento teórico ni empírico). La segunda hipótesis plantea que el apoyo social sirve de protector o amortiguador del distrés o efectos negativos generados por los eventos vitales estresantes. Este planteamiento manifiesta que el efecto rehabilitador del apoyo social tiene dos vías de acción fundamentales:

- La intervención de apoyo social entre el evento estresor y/o sus expectativas y la situación estresante como tal, a través de la disminución o prevención de la respuesta de estrés, lo que favorece la respuesta eficaz ante la amenaza o su enfrentamiento adecuado.

- La intervención del apoyo social entre el estrés y sus consecuencias patológicas a través de medidas para reducir la percepción del estrés o comportamientos que influyan de manera directa en la enfermedad y los procesos patológicos.

Actualmente se considera que estos enfoques no son excluyentes, sino que el apoyo social visto de manera sistemática engloba efectos protectores directos y amortiguadores contra la enfermedad o malestar. Marín (2002) refiere que el sistema de apoyo social es importante pues permite el mantenimiento de la integridad psicológica y física de la persona a lo largo del tiempo y que sus funciones primarias son aumentar las capacidades personales de sus miembros y promover el logro de sus metas vitales.

Igualmente, se ha encontrado que los pacientes con diversas enfermedades crónicas que tienen un alto nivel de satisfacción en relación al apoyo social presentan un mejor grado de 
adaptación a la enfermedad y es más importante la percepción que tiene el paciente de lo adecuado del soporte social que el soporte que realmente recibe. La percepción de disponer de apoyo protege a las personas de los efectos patogénicos de los eventos estresantes.

\section{MIRADA DEL SOPORTE SOCIAL DESDE DISTINTAS DISCIPLINAS.}

El interés del estudio de la influencia del apoyo social sobre la salud y la enfermedad ha aumentado durante las últimas décadas, la bibliografía al respecto es muy amplia. Las investigaciones respaldan el estudio del fenómeno desde tres perspectivas: Estructura de la Red (Lazos básicos entre miembros de la red social), Funciones de Apoyo (funciones de la fuente de apoyo que fluyen a través de estos lazos de la red), y la naturaleza de las relaciones (contacto de los individuos con los integrantes o miembros de la red).

Se ha encontrado que el apoyo social ayuda a las personas con enfermedades crónicas a reducir el estrés en la vida (Eldridge, 1994; Kimberly y Serovich, 1999), provee un espacio para relajarse y desarrollar un compartir positivo. Esto, a su vez, reduce los efectos negativos de la condición de salud. Algunos estudios han reportado que el apoyo social tiene un impacto positivo en el desarrollo de autoeficacia, definiéndola como la creencia individual en la habilidad y competencia para realizar alguna conducta en particular (Penninx, van Tilburg, Boeke, Deeg, Kriegsman y van Eijk, 1998).

Según la revisión de los trabajos realizados por Ren y cols. citados por Díaz y García, el apoyo social disminuye los riesgos de problemas psicológicos y de enfermedades psiquiátricas. Otros autores revelan en sus estudios la diferencia entre la calidad y cantidad del apoyo social, de tal forma que la calidad del apoyo es un mejor predictor de la autopercepción que la cantidad.

Estudios relacionadas con los niveles de apoyo social y el VIH/SIDA han revelado claramente que las personas con mayores índices de apoyo social presentan menos probabilidades de experimentar emociones disfóricas, lo que se asocia con un mejor estado de salud (Hays, Turner y Coates, 1992). Se ha encontrado que los mayores niveles de satisfacción con el apoyo social se observan principalmente en personas que participan activamente en grupos de autoayuda y en redes sociales de personas seropositivas (Barroso, 1996; Fasce, 2001; Kadushin, 1999).

\section{SOPORTE SOCIAL DESDE LA ENFERMERÍA}

Desde la perspectiva de la enfermería la revisión del estado del arte en la temática identifican a Norbeck, enfermera investigadora en apoyo social desde la década de los 70, pionera con sus aportes conceptuales e instrumentales en esta temática. Presenta una reseña de investigaciones conducidas por enfermeras sobre apoyo social, y en búsqueda bibliográfica encontró 67 publicaciones escritas por enfermeras, de las cuales 53 representaban estudios sobre la temática

Las investigaciones con poblaciones clínicas de la práctica de enfermería relacionados con el apoyo social, se han categorizado en cuatro áreas: Cambios de vida, desempeño del rol, comportamientos en salud y crisis o conductas de enfermedad; los cuales han reportado aspectos significativos del apoyo social en diferentes situaciones del proceso vital humano.

De los cambios de vida de interés para enfermería el apoyo social se ha estudiado en las etapas del embarazo y el envejecimiento. En esta área los estudios realizados confirman 
que el apoyo social es importante con relación al desarrollo de complicaciones, salud física o psicológica de los padres en espera y adaptación a la paternidad.

Respecto al envejecimiento, el apoyo social se ha estudiado con relación al cuidado en casa, la institucionalización, y la salud física y psicológica; destacándose en los resultados la familia como principal recurso de apoyo social del anciano para el cuidado en casa, y su ausencia como factor determinante en la institucionalización del adulto mayor, igualmente resaltan la valoración del apoyo social por parte de las personas mayores como elemento relacionado con la salud psicológica más que en la salud funcional o física.

Sobre el desempeño del rol se ha explorado con relación a los roles familiares y del trabajador principalmente en personas del género femenino. En cuanto a los roles familiares, se ha estudiado el apoyo social en diferentes situaciones, entre ellas los papeles del familiar que brinda cuidado a un anciano, esposa o esposo enfermo, específicamente el bienestar físico, psicológico y social de personas mencionadas como principal fuente de ayuda de padres ancianos de pacientes con infarto del miocardio, destacándose entre sus resultados la unión familiar como fuente de bienestar psicológico, y los parientes, vecinos, enfermeras registradas y médicos, se identifican como principales fuentes de apoyo. Torres et al ( 2008) al revisar la evidencia científica disponible sobre la efectividad de distintos programas e intervenciones dirigidas a los cuidadores informales en España, encontraron que las enfermeras son los profesionales que proponen más estudios de intervención o de soporte a los cuidadores, desempeñando un importante papel en el equipo multidisciplinar, al proporcionar cuidados formales tanto a las personas dependientes como a sus cuidadores familiares.

En Colombia, se citan algunos estudios descriptivos y correlaciónales en la temática. Los estudios descriptivos se han enfocado en su significado, los estudios correlaciónales han explorado las variables asociadas al soporte social. Los datos arrojados muestran que la familia es la mayor proveedora de apoyo social, la red con que cuentan estos pacientes es estable y duradera, siendo el poyo emocional representado en afecto, afirmación y ayuda tangible el más importante, lo que les permite asumir una actitud positiva ante la enfermedad crónica.

\section{CONCLUSIONES}

El estudio del soporte social se está convirtiendo en una importante variable de análisis en la practica de enfermería, especialmente en la búsqueda de estrategias de intervención ante problemas de salud con implicaciones tan complejas como las enfermedades crónicas.

El concepto de soporte social se operacionaliza en tres categorías amplias: Redes sociales, soporte social percibido, y soporte social efectivo. La primera se refiere a las conexiones que los individuos tienen para significar a otros en su medio social, teniendo un sentimiento psicológico de comunidad. La segunda es la valoración cognitiva que hace la persona respecto a quiénes le pueden proveer determinada ayuda en momentos de necesidad; y el soporte social efectivo representa las acciones soportivas mismas, a la ayuda efectiva y las acciones que recibe de los otros en momentos de necesidad.

Las redes de apoyo social juegan un importante papel en el bienestar de los cuidadores, protege contra el estrés generado por la enfermedad y capacita al paciente para reevaluar la situación y adaptarse mejor a ella, ayudándole a desarrollar respuestas de afrontamiento. 
La familia es la fuente principal de apoyo social con que cuenta el enfermo crónico para afrontar con éxito los problemas a que da lugar la enfermedad, destacándose el papel de la cuidadora primaria, quien aporta el máximo apoyo instrumental, afectivo y emocional.

Las investigaciones realizadas respecto al efecto de intervenciones y programas de soporte y apoyo social al enfermo crónico y su cuidador documentan resultados positivos con relación al desarrollo de mecanismos de afrontamiento, control del estrés generado por los efectos de la enfermedad o la sobrecarga del cuidador y disminución de la ansiedad, depresión y aislamiento que acompañan la experiencia de vivir en situación de enfermedad crónica.

\section{REFERENCIAS BIBLIOGRÁFICAS}

1 Organización Mundial de la Salud. Prevención y control de las enfermedades no trasmisibles: Aplicación de la estrategia mundial. Informe de la Secretaria. 61aㅗ Asamblea Mundial de la Salud. Abril 2008. P. 3

2 OMS. Chronic disease and health promotion, [fecha de consulta Julio 2007].Disponible en: http://www.who.int/chp/chronic_disease_report/part//es/index $4 \mathrm{html}$.

3 THE WORLD BANK. Disease Control Priorities Project. Enfermedades no trasmisibles. [Citado en Julio 2006]. P.1 Disponible en: www.dep 2.org

4 Lucumi, Diego, Gutiérrez, Alejandra, Moreno, José et al. Planeación Local para enfrentar el Desafío de las Enfermedades Crónicas en Pasto, Colombia. Rev. Salud pública, mar. /mayo 2008, vol.10, no.2, p.343-351.

5 Horton R. The neglected epidemic of chronic disease. Lancet 2005.p 366(9496):1514

6 MINISTERIO DE LA PROTECCION SOCIAL. Decreto № 3039 de agosto de 2007. Plan Nacional de Salud Pública 2007 - 2010.

7 Moreno María Elisa. Discapacidad en América Latina: Una visión de enfermería. El arte y la ciencia del cuidado, Grupo de cuidado de Enfermería, Unibiblos, Universidad Nacional de Colombia. 2002, p.15

8 ORGANIZACION MUNDIAL DE LA SALUD. Preparación de los profesionales de la salud para el siglo XXI, el reto de las enfermedades no transmisible y salud mental enfermedades crónicas y promoción de la salud s.I; OMS, 2005. 15t.

9 ORGANIZACIÓN PANAMERICANA DE LA SALUD. La salud en la Américas. IV. Promoción de la salud en las Américas. Edición 2002. Volumen 1. P.193

10 DANE, Departamento Administrativo Nacional De Estadística. Censo General 2005 personas con discapacidad permanente Bogotá D.C. Septiembre 8 de 2006.

11 DANE Departamento Administrativo Nacional De Estadística. Censo General 2005 personas con discapacidad permanente. Cúcuta 2005.

12 Mendoza Tarazona Martha, Ureña Molina María del Pilar, Villamil Santander Wilmer. Efecto de un programa educativo en la habilidad de cuidado de los cuidadores en el Centro de Rehabilitación Cardioneuromuscular, Revista Ciencia y Cuidado, Volumen 5 Número 1 Enero-Diciembre 2008, Facultad Ciencias de la salud, Universidad Francisco de Paula Santander, p. 16

13 Fuentes González N, Ojeda Medina AA. Soporte y red social en el adulto maduro con Hipertensión Arterial y/o Diabetes Mellitus tipo II en riesgo de insuficiencia renal. Biblioteca Lascasas, 2007; 3(3). Disponible en: http://www.indexf.com/lascasas/documentos/lc0261.php

14 Pinto Afanador, N., Barrera Ortiz, L., \& Sánchez Herrera, B. 2006 Jun 16. Reflexiones sobre el cuidado a partir del programa "Cuidando a los cuidadores". Aquichan [Online] 5:0. Disponible: http://biblioteca.unisabana.edu.co/revistas/index.php/aquichan/article/view/142 
15 De los Ríos JL., Sánchez JJ., Barrios P., Ávila TL. Quality of life in patients with diabetic nephropathy. Invest educ enferm 2005; 23(1): 34-41.

16 Schwarz Karen A, Roberts Beverly. El apoyo social y la tensión de los cuidadores familiares de adultos mayores. Nursing Practice In Action. Vol. 14 (2), January 2000. P 79 $(77-90)$

17 Achury Saldaña Diana Marcela. Autocuidado y adherencia en pacientes con falla cardiaca. Revista Aquichan, año 7, Vol. $7 \mathrm{~N}^{\circ} 2$, Fa cultad de Enfermería, Universidad de la Sabana, p. 142

18 Lemos Giráldez Serafín, Fernández Hermida José Ramón. Revista Psicothema, 1990. Vol. 2, no 2, Facultad de Psicología de la Universidad de Oviedo, pp. 113-135

19 Kaplan, J. R., Manuck, S. B., Clarkson, T.B., Lusso, F.M., Taub, D.M. y Miller, E.W. (1983). Social stress and atherosclerosis in normocholesterolemic monkeys. Science, 220, 733-735.

20 Díaz Heredia Luz Patricia, Soporte social en situaciones de enfermedad crónica, La investigación y el cuidado en América Latina, Unibiblos, primera edición, Universidad Nacional de Colombia, p 323

21 Saranson I. G., Saranson, B. R., Hacker, T. A. y Bashman, R. B. (1985). Concomitants of social support: Social skills, physical attractiveness and gender. Journal of Personality and Social Psychology, 49, 469-480.

22 Muñoz de Rodriguez Lucy. El apoyo social y el cuidado de la salud humana. Cuidado y práctica de enfermería, Unibiblos Facultad de Enfermería, Universidad Nacional de Colombia, Santa Fe de Bogotá, p 99

23 Torres Uria Margarita Ruth, Estudio comparativo en pacientes con tuberculosis pulmonar frotis positivo nunca tratados, antes tratados y Crónicos en sus niveles de depresión y soporte social, Tesis Universidad Nacional Mayor de San Marcos. Facultad de Psicología. Escuela de Post-Grado, 2002, Disponible en: http://sisbib.unmsm.edu.pe/BibVirtualData/Tesis/Salud/Torres_U_M/cap2.pdf.

24 Finfgeld-Connett, Clarification of Social Support, Journal of Nursing Scholarship, 2005; 37:1,4-9.

25 Pastor, M; López-Roig, S; Rodríguez-Marín, J; Salas, E; Sánchez, S. y Terol, M. (1994). El papel del apoyo social en la experiencia de dolor crónico. Revista de Psicología Social. 9, 179-191.

26 Roca M A, Pérez M. Apoyo social: su significado para la salud humana. Ciudad Habana. Editorial Félix Varela. 1999

27 García Martín MA. Beneficios del apoyo social durante el envejecimiento: efectos protectores de la actividad y el deporte. [En línea] 2000; URL Disponible en: http://www efdeportes. com

28 Rodríguez Marín J. Apoyo social y salud. En: Guía de Estudio y Bibliografía Básica Módulo Psicología, Salud y enfermedad. Maestría Psicología de la Salud. Ciudad Habana: Escuela Nacional de Salud Pública; 2002.p. 94-103

29 Vinaccia, Stefano, Margarita Quinceno, Japcy, Zapata, Carla. Calidad de vida relacionada con la salud y emociones negativas en pacientes con diagnóstico de enfermedad pulmonar obstructiva crónica (EPOC). Psicol. Caribe, dez. 2006, no.18, p.89108. ISSN 0123-417X.

30 Lemos Giráldez Serafín, Fernández Hermida José Ramón, En revista Psicothema, Op.cite 115

31 Fuentes González N, Ojeda Medina AA. Soporte y red social en el adulto maduro con Hipertensión Arterial y/o Diabetes Mellitus tipo II, Op.cite. p 48

32 Díaz Sánchez V, García Huete ME, Marín Morales MD, Campo Cecilia E. Los intercambios de apoyo en el cuidado de un aborto espontáneo. Biblioteca Lascasas, 2008; 4(1). Disponible en <http://www.index-f.com/lascasas/documentos/lc0306.php 
33 Toro Alfonso, José; Varas Díaz, Nelson; Núñez, Júnior. Las redes de apoyo social para las personas que viven con VIH/SIDA en Puerto Rico: Del aislamiento a la plena ciudadanía mediante la acción social. En publicación: Revista de Ciencias Sociales 13. CIS, Centro de Investigaciones Sociales, Facultad de Ciencias Sociales, Universidad de Puerto Rico, San Juan, Puerto Rico. Diciembre.2004. Disponible en: http://bibliotecavirtual.clacso.org.ar/ar/libros/pr/cis/rcs/13/redes.pdf

34 Muñoz de Rodríguez Lucy. El apoyo social y el cuidado de la salud humana, Op.cite, 102 35 Norbeck, Janes. Apoyo Social. Capítulo 4. Escuela de Enfermería Universidad de California, San Francisco. 2001. P 9

36 Langer A. Apoyo durante el embarazo a mujeres con mayor riesgo de recién nacidos de bajo peso al nacer: Comentario de la BSR (última revisión: 2 de octubre de 2003). La Biblioteca de Salud reproductiva de la OMS; Ginebra: Organización Mundial de la Salud.

37 Alvarado M, Rubén, Medina L, Ernesto y Aranda $\mathrm{CH}$, Waldo. El efecto de variables psicosociales durante el embarazo, en el peso y la edad gestacional del recién nacido. Rev. Méd. Chile, mayo 2002, Vol.130, No.5, p.561.

38 Zea MC, Torres BP. Adultos mayores dependientes hospitalizados: la transición del cuidado. Invest Educ Enferm. 2007; (25)1: 40-49.

39 Calvete, Zumalde Esther. Apoyo Social y Síntomas psicológicos en ancianos institucionalizados. Revista Española de Geriatría y Gerontología, 29(2), 73-78.

40 Norbeck, Janes. Ibíd. P 9 -13

41 Norbeck, Janes. Social support. Annu Rev Nurs Res. 1988; 6:85-109.

42 Torres Egea M ${ }^{a}$ Pilar, Ballesteros Pérez Esperanza, Sánchez Castillo Pablo David. Programas e intervenciones de apoyo a los cuidadores informales en España. Gerokomos. [Periódico en la Internet]. 2008 Mar [citado 2008 Dic 23]; 19(1): 9-15. Disponible en: http://scielo.isciii.es/scielo.php

43 Camacho Parrada, Nelsy Yaneth Caracterización del soporte social en gestantes adolescentes asistentes a las IPS popular y recreo, entre julio-agosto-septiembre de 2000, Revista Avances de enfermería, Vol. XIX No 2 de 2001, Facultad de enfermería, Universidad Nacional de Colombia p. 23

44 Piñeros Serrada Blanca Estella, Características del soporte social recibido por madres primíparas que lactan y las que no lactan exclusivamente a su hijo. Revista Avances de enfermería, Vol. XIX No 2 de 2001, Facultad de enfermería, Universidad Nacional de Colombia, p. 32

45 Abaunza de González M, Acosta S, Álzate Posada ML, Amaya P, Barbosa G, Barrera L, et al. La investigación y el cuidado en América Latina. En: Natividad Pinto A, compiladora. Comprender la situación de cronicidad que viven nuestros pacientes, Bogotá: editorial unibiblios; 2005. p 311.

46 Barrera O. L, Galvis L. CR, Moreno F. ME, Pinto A. N, Pinzón R. ML, Romero G. E, Sánchez H. B. La habilidad de cuidado de los cuidadores familiares de personas con enfermedad crónica. Invest. educ. enferm 2006; 24(1): 36

ISSN 1695-6141

@ COPYRIGHT Servicio de Publicaciones - Universidad de Murcia 